\title{
DEL ENFOQUE EMIC A LOS PROCEDIMIENTOS CRÍTICOS DE INTRERPRETACIÓN. RETROSPECTIVA Y ANTICIPACIONES.
}

\author{
Aurora GONZÁLEZ ECHEVARRÍA ${ }^{1}$
}

\begin{abstract}
Resumen
El propósito en este artículo es fundamentar la crítica a una forma de concebir el conocimiento antropológico, que lo caracteriza por la contraposición entre los enfoques emic y los enfoques etic, como caso particular de contraposición entre enfoques hermenéuticos y científicos. Si se aplica a la Antropología la concepción estructural de las teorías y se acepta la corregibilidad de las interpretaciones de significados, todas las proposiciones que contienen las etnografías, tanto las relativas a datos como a las relaciones que se establecen entre ellos tienen el mismo carácter científico, al tiempo que comparten las cautelas que apliquemos al término. Las dicotomías no sólo resultarían inadecuadas, sino carentes de sentido. Finalmente se esbozan las consecuencias de esta propuesta metodológica sobre la forma de pensar la comparación transcultural.

Palabras clave: Conocimiento antropológico, dicotomía etic/emic, antropología científica, antropología hermenéutica, concepción estructural de las teorías, monografías etnográficas, comparación transcultural.
\end{abstract}

\begin{abstract}
.
The aim of this article is to provide a basis for the critique of a certain way of understanding anthropological knowledge which was characterized by the contraposition between emic and etic elements as a particular case of contraposition between hermeneutical and scientific insights. If the structural conception of theories is applied to Anthropology and if we do accept the possibility of correcting the interpretation of meanings, all propositions contained in ethnographies, both those concerning data as those concerning the relationships established among them, have the same scientific character, and share the cautions which we may apply to the term. Thus, dichotomies not only would be inadequate, but also meaningless. Finally, I sketch the consequences of such a methodological proposal upon the way of thinking about cross-cultural comparison.
\end{abstract}

Keywords: Anthropological Knowledge, Emic/Etic Dichotomy, Scientific Anthropology, Hermeneutical Anthropology, Structural Conception of Theories, Ethnographic Monographies, Cross-Cultural Comparison.

\section{Résumé}

Cet article a pour but fonder la critique sur une façon de concevoir la connaissance anthropologique, qui caractérise cette connaissance en utilisant l'opposition entre les approches emic et etic, au cas particulier de l'opposition entre approches herméneutiques et scientifiques. L'application à l'anthropologie de la conception structurelle des théories et $\mathrm{l}^{\prime}$ acceptation de la possibilité de corriger $1^{\prime}$ interprétation des signifiances, donne le même caractère à toutes les

\footnotetext{
${ }^{1}$ GETP/GRAFO - Dpto de Antropología Social y Cultural - Universidad Autónoma de Barcelona

El GETP -Grupo de Estudio Transcultural del Parentesco- es un equipo de investigación de la UAB forma parte del GRAFO -Grup de Recerca en Antropologia Fonamental i Orientada. El GRAFO ES un GRC de la Generalidad de Cataluña, que coordino. Se recogen aquí parte de los resultados del proyecto del MCYT "Teoría transcultural de la reproducción de los grupos humanos" (SEJ2006-10864)

C.E: Aurora.Gonzalez@uab.cat
}

Fecha de recepción del artículo: Octubre 2009

Fecha de evaluación: Noviembre 2009 
propositions que font partie des ethnographies, soi celles relatives aux donnés comme celles qui proposent des rapports parmi eux, avec les précautions qu'on doit prendre avec le terme scientifique. Les dichotomies seraient non seulement inadéquates, mais aussi dépourvues de sens. Pour finaliser, on esquisse les conséquences de cette position méthodologique pour la caractérisation de la comparaison transculturelle.

Mots-clé: Connaissance anthropologique, dichotomie etic/emic, anthropologie scientifique, conception structurelle des théories, monographies ethnographiques, comparaison transculturel.

Marilyn Strathern, en un brillante postfacio a Gringrich y Fox, 2002, eds., sostiene que e el paso de los métodos comparativos clásicos ( CM1), basados en una concepción científica fuerte de la Antropología a nuevos métodos comparativos (CM2) que tienen más en cuenta su responsabilidad pública, es una reminiscencia del paso del Mode 1 al Mode 2 de producción de conocimiento científico, tal como Gibbons et alli, 1994 identifican 1 por referencia a cambios en el entorno de la producción de ciencia, entre ellos la heterogeneidad del soporte institucional, las localizaciones multisituadas, y la responsabilidad social. ${ }^{2}$

Haciendo un uso laxo de esta consideración sobre cómo los procedimientos de comparación dependen de las formas de conceptualizar el conocimiento científico, puedo decir que mi propósito en este artículo es contribuir al paso de una forma de concebir el conocimiento antropológico, caracterizado por la contraposición entre los enfoques emic y los enfoques etic, como caso particular de contraposición entre enfoques hermenéutico y científico, a otra forma donde estas dicotomías pierden su sentido, y esbozar las consecuencias de esta propuesta metodológica sobre la forma de pensar la comparación transcultural.

En 1987 publiqué un libro derivado de mi tesis doctoral, La construcción científica en Antropología, que calificaría ahora de cientifista no porque abrazara ningún positivismo sino porque sostuve en él que las antropologías científica y hermenéutica eran alternativas. Poco después participé en un encuentro entre antropólogos para el que se me pidió una comunicación sobre la Antropología como ciencia fuerte. Lo que sostuve fue que más bien todas las ciencias eran débiles, interesada en aquel momento por la imposibilidad de establecer un criterio de demarcación preciso entre conocimiento científico y no científico y por la imposibilidad de comparar entre si con criterios estrictamente lógicos matrices disciplinares distintas (Kuhn, 1969) o distintos programas de investigación en el sentido de Lakatos, 1975a,

En aquel encuentro, Rivière pronunció una conferencia en la que sostuvo que, desde que Winch escribió en 1959 The Idea of a Social Science, era impensable una antropología científica. Volví a casa con el convencimiento de que tenía razón en mi defensa de un antropología científica, utilizando el término "científica" con todas las precauciones críticas, pero que tendría que trabajar mucho más para fundamentar mis convicciones.

\footnotetext{
2 "Take the charge of the "hard-core scientific nature" of CM1, for instance. The move from CM1 to CM2 is nothing if not reminiscence of the move from the Mode 1 to Mode 2 type of knowledge production that Gibbons and his collegues (1994) have identified from within science. Scientific practice now faces new pressures to take social context into account. Of course, Gibbons et al. are talking about knowledge production rather than about methods or models, and thus about practices ( heterogeneity of institutional support, multisituated locations, social accountability and reflexive engagement wiyh non.scientist) that change not the procedures but the environment of scientific work. Nonetheless, the "growth of uncertainty " to which they subsequently refer ( Nowotny et al. 2001) is also one of the comtemporary context for the approach we shall see advocated by Gringrich and Fox" ( p. XIV).
} 
No se que me llevó a empezar por un análisis detallado de la historia de la dicotomía emic/etic, cuando en la Antropología española de finales de los 80 las palabras clave eran "hermenéutica" y "descripción densa". Tal vez el interés que tenía entonces por el análisis de las terminologías de parentesco y por los debates de la llamada en los 60 "nueva etnografía" sobre la validez semántica y estructural de las interpretaciones que hacían los antropólogos de las terminologías.

Aunque más interesada en el concepto de programa de investigación de Lakatos que en el de revolución científica de Kuhn, parecía evidente la carga cultural de las teorías científicas que Kuhn trató de recoger primero con el concepto de paradigma y después de matriz disciplinar. Mi preocupación estaba centrada en la especificidad de la ciencia como subcultura específica. En las diferencias entre los procesos que podían llevar a que en una cultura se desarrollara una terminología de parentesco determinada - ahora diría una forma de categorización social-y los procesos que podían llevar a un antropólogos a atribuir el uso de una terminología específica a los miembros de una determinada sociedad. Nunca pensé esa diferencia en términos de emic frente a etic. Pero si consideré que un conocimiento detallado de la génesis y la historia de la dicotomía podía proporcionar respuestas a mis preguntas y fundamento a mis convicciones.

Trabajé sobre la dicotomía emicletic en algunos cursos del programa de doctorado, tras darme cuenta pronto de que efectivamente era Pike el predecente inmediato de penetrar de manera más profunda en la diversidad cultural. Ante una diferenciación que siempre me pareció confusa, pasé así del asombro ante la rotundidad con la que se adjudicaba a una afirmación el adjetivo de "emic" o "etic"- como por lo demás se sigue haciendo- a constatar que efectivamente eran muy ricos los problemas que discutían los autores verdaderamente interesados en la dicotomía. A mediados de los 90 había llegado a las siguientes conclusiones:

- la ambigüedad en la utilización de los términos emic y etic empieza en Pike, que oscila entre considerar el enfoque etic externo cuando lo piensa como intercultural, comparativo y preliminar cuando lo caracteriza como el bagaje integrado por las unidades y clasificaciones que el antropólogo aprende en su periodo de formación

- Sólo se puede entender el enfoque etic como preliminar e hipotético, como el equipaje conceptual que permite el acceso al sistema, como el punto de partida de un análisis que se va refinando progresivamente hasta que la presentación final se hace en unidades emic, pertinentes para la cultura estudiada.

- Pike encuentra dificultades para seguir con la analogía entre la lengua y el resto de la cultura al no ser capaz de precisar cuales serían las unidades en las que debería hacerse una eventual descripción etic. Más claro es respecto a la descripción emic: trataría de dar cuenta de las estructuras descubiertas a las que se llega a través de las verbalizaciones de los nativos pero también, y sobre todo, a través de la observación de reacciones provocadas intencionadamente y de la observación de comportamientos significativos. No existe en Pike una preocupación teórica por la causa de esas estructuras de manera que el bagaje previo parece más tipológico que teórico.

- Pike no sitúa la validez de los enfoques emic en el acuerdo de los nativos, como más tarde le atribuyó Harris: era muy consciente de los fenómenos de falsa conciencia. Goodenough, que hizo el desarrollo más interesante de Pike, habló en 1956 a de conceptos del mundo cognitivo truk no porque los truk los vieran así sino porque operaban así, anticipándose a la distinción que establecieron Wallace y Atkins, 1960, entre la validez psicológica de las terminologías (lo que es real para el nativo) y la validez estructural (lo que es real para el antropólogo). Pero fue Hammel quien realizó la aportación más potente al debate: los análisis semánticos serían modelos del antropólogo que tratan de aprehender el sistema nativo. 
- En 1964 Harris adopta una forma absolutamente distinta de aproximarse a los hechos de la cultura: estudiar los comportamientos no verbales y, aun más, los elementos en principio puramente físicos de estas conductas. Apuesta por un operacionalismo que se centre en los movimientos corporales y sea fácilmente replicable, de manera que observadores independientes realizando las mismas operaciones puedan llegar a resultados similares. Pero pronto se ve el límite de este enfoque, porque no es posible, por ejemplo, definir la "residencia patrilocal" partiendo de que el padre es el hombre cuyo semen fecundó el óvulo de la mujer por cuya vagina salió a la luz el sujeto residente. Muchos siglos antes de la edad de las Nuevas Tecnologías Reproductivas habría sido ya una definición inapropiada ya para el derecho romano.

- Aunque Harris abandonó pronto la búsqueda de un lenguaje fisicalista que permitiera describir las conductas humanas en términos de los movimientos corporales y su orientación espacial, mantuvo desde 1964 lo esencial de su lectura de Pike: que el punto de vista emic sería subjetivo, orientado al sujeto, y el punto de vista etic intersiubjetivo, orientado a la comunidad de los antropólogos (Harris, 1964: 137 y s.).

- Fue Goodenough, en 1956 b en su artículo sobre las reglas de residencia truk y de manera sistemática en 1970, en el capítulo 4 de Description and Comparison el que propuso la concepción de un lenguaje etic que se acerca más a los objetivos de la fonética general definidos por Saussure: conocer y clasificar los elementos con los que una lengua opera (Saussure, 1965 (1915): 94).

- Goodenough, interesado en la comparación transcultural, no piensa, como Harris, en un lenguaje etic hecho de movimientos físicos y opuesto al emic, sino que considera que el lenguaje etic se elabora a partir del las unidades emic, es decir, a partir de los conceptos que el etnógrafo tiene que introducir para dar cuenta de las distinciones significativas en distintos ámbitos de distintas culturas (recordemos: no significativas porque los nativos las vean así , sino porque así operan). A medida que más aspectos de más culturas son estudiadas en profundidad más rico se hace el bagaje etic disponible para cualquier etnógrafo que se aproxima al estudio de una nueva cultura.

Los puntos que anteceden estaban contenidos en una comunicación que presenté a un congreso en 1996, y habían sido extraídos de un borrador ya en aquel momento bastante completo del libro que publiqué en 2009. Siguen siendo válidos como resumen de estas tesis recientes. Para que fuera más completo tendría que recoger la progresiva sofisticación de la visión de Harris de la dicotomía, que nada pudo contra la visión canónica que él formuló en 1968 y 1971 a través de dos libros que fueron muy leídos, su historia de la Antropolologia y su introducción general: desde entonces por emic se entienden generalmente los modelos nativos y por etic los del antropólogo. También tendría que hacer referencia, un resumen más completo, al resultado de mis intentos, bastante recientes, de diferenciar conceptos descriptivos, aproximativos, interpretativos y teóricos. Pero ninguno de los dos desarrollos afecta al núcleo de la argumentación.

Si me pregunto por qué no publiqué este trabajo hasta muchos años después, más allá de los retrasos editoriales, creo que la respuesta está también en el artículo de 1996. Me planteaba entonces, a propósito de los análisis semánticos de las terminologías de parentesco, cómo poner a prueba nuestras hipótesis sobre los modelos cognitivos indígenas con descripciones de estructura también nuestras y anticipaba mi interés en escapar a la circularidad elaborando constructos que incorporasen cogniciones, por medio de descripciones de estructura que fueran hipótesis sobre cogniciones y comportamientos (p.121). Y en las conclusiones explicitaba lo que constituía en el ámbito de los métodos de investigación mi proyecto de trabajo: reformular los procedimientos de construcción teórica otorgando en ellos un papel predominante y renovado a la concepción estructural de las teorías y a la comparación intercultural. 
En los años siguientes, trabajé en esta línea hasta concluir con lo que considero un desarrollo en espiral que empieza con la antropología científica clásica, se aproxima a al hermenéutica desde Dilthey a Gadamer, y se extiende sobre los proyectos interpretativos en Antropología y sobre la complejidad del conocimiento científico hasta concluir con la defensa de un conocimiento antropológico científico que integre en sus explicaciones interpretaciones y relaciones y que indague desde una epistemología crítica sobre sus límites y sus condicionantes e implicaciones sociales para tratar de controlarlos ( González Echevarría, 2003)

Desde la perspectiva del método dos fueron las herramientas fundamentales para llegar a estas conclusiones. La primera fue la aplicación a las etnografías de la concepción estructural de las teorías. La segunda la diferenciación entre dos desarrollos o mejor aún entre dos componentes de la hermenéutica en el siglo XX: el ontológico y el metodológico o procedimental.

En los años 60 Suppes $(1962,1966)$ propuso una nueva metateoría que hiciera frente a los problemas de la que desde entonces se llama concepción heredada (axiomático-deductiva) de las teorías: entre otros la dificultad para establecer la correspondencia entre términos teóricos y observacionales, la imposibilidad de establecer criterios de sencillez o probabilidad lógica que permitiera comparar las teorías y la carga teórica de la percepción y de la selección de los datos.

Para Suppes, una teoría es una estructura que puede atribuirse a conjuntos de fenómenos o individuos; que se predica de conjuntos de fenómenos. Entendiendo por estructura las relaciones entre los elementos de un conjunto y las propiedades de estas relaciones, formular una teoría es atribuir a un conjunto una estructura determinada.

Si pensamos en una teoría como una estructura $S$, hay una serie de objetos $M$ que son los modelos de $S$ y que constituyen la extensión del $S$, donde " $M$ es un $S$ " si tiene la estructura $S$. Si un fenómeno aparece en distintos modelos, sus propiedades deben de tener en todos el mismo valor, son las que Sneed denomina condiciones de ligadura (de los modelos). Los modelos y las condiciones de ligadura constituyen el núcleo de la teoría. Su base empírica, o dominio de aplicaciones $A$, son las porciones de la realidad que se conceptualizan como compatibles con el núcleo, es decir, que se piensan como posibles aplicaciones de la teoría y están constituidas por clases de conjuntos: números, sistemas planetarios, sociedades con brujería del vientre.

Pero no basta el dominio de aplicaciones, o marco empírico, para identificar el componente empírico de las teorías. En cada momento parte de estas aplicaciones son aplicaciones realmente propuestas. Algunas de las aplicaciones propuestas pueden cambiar con el tiempo pero las aplicaciones paradigmáticas, los modelos que hacen que una teoría sea propuesta, y se acepte, están en la formulación inicial de la teoría y continúan siendo parte de la teoría durante toda su historia..

Esta concepción parece especialmente adecuada para la etnografía. Un ejemplo de aplicación a casos etnográficos, podría ser la estructura de linajes segmentarios tal como la definió EvansPritchard en 1940 en su estudio sobre los nuer, con los linajes mínimos equivalentes, las reglas de fusión/fisión, la correspondencia entre los segmentos del sistema de linajes y las secciones del sistema territorial, las figuras carismáticas que median en los conflictos entre linajes y permiten que no se fracture la tribu. En cuanto a su dominio de aplicaciones, pensemos en su aplicación a un buen número de sociedades del África subsahariana y en el fracaso de los intentos de aplicación a las sociedades del Pacífico. Pensar las etnografías como predicados de estructura nos lleva a considerar que en ellas se formula la teoría antropológica y a preguntarnos cuales son los datos que entran en relación en estos predicados y cual es el estatuto cognoscitivo de las interpretaciones de los significados.

Cuando hablo de la Antropología hermenéutica no pienso en esa hermenéutica utens presente en buena parte de las etnografías escritas a lo largo del S. XX, y aún anteriores, que nos trasmiten y 
nos proporcionan la comprensión de una cultura. Me refiero a las declaraciones programáticas que se inspiran en Gadamer, 1960 y particularmente en aquellas ideas de Gadamer que tienen mayores implicaciones existenciales, como la que expresó en 1953 sobre la sujeción al método, que impediría conocer muchas verdades porque la ciencia termina en las situaciones límite en que la comunicación no es la trasmisión de conocimientos mediante pruebas categóricas sino el trato de una existencia con otra ( 1994 a (1953): 44). Es este Gadamer el que está detrás del énfasis en la alteridad, en la insistencia en que la tarea fundamental de la hermenéutica es el retorno a si mismo desde el ser del otro (1993 (1960): 43), en la comprensión como fusión de horizontes ( 1994 b ( 1957): 61), (1993 (1960): 372, 376).

Coincido que esta es la mayor aportación de la Antropología pero ¿qué es comprender? Gadamer escribió en 1985 que comprender es ya interpretar, o, en palabras de Schleiermacher (autor en el que basó Dilthey su fundamentación hermenéutica de las ciencias sociales), "el interpretar sólo difiere del comprender como el habla en voz alta del habla interior" (1994 c (1985): 26). Así pues, la pregunta se convierte en ¿qué es interpretar? Y no es una pregunta retórica si atendemos a lo que Gadamer constata escribe en 1977 , que

\begin{abstract}
"[muchos] sobre todo desde que la hermenéutica se ha convertido en palabra de moda y a cualquier "interpretación" se llama hermenética, abusan del término y su significado e invierten el sentido para el que se utilizó el término: ven en la hermenética una nueva metodología para legitimar en realidad la intransparencia del método o el encubrimiento ideológico" ( 1994d, (1977: 390).
\end{abstract}

Tal vez esta postura anti-metodológica fue estimulada por el propio Gadamer, que tenía una concepción de la ciencia, de la verdad de la ciencia, que ya llevaba tiempo haciendo crisis cuando escribe, pero no fue este el mayor problema de quienes lo invocaron en Antropología sino el no tener en cuenta que esa interpretación que lleva a la ampliación y fusión de horizontes también tenía sus propias reglas. Y de ellas también habló Gadamer en 1959, cuando sostuvo que es el análisis existencial de Heidegger donde la estructura circular de la comprensión adquiere su verdadero significado. Y escribe: "Heidegger reconoce (...) que la comprensión del texto está determinada permanentemente por el movimiento anticipatorio de la precomprensión “(Gadamer, 1994e(1959):67).

Pero no se detiene en ella. Entender un texto, un momento, supone siempre un proyecto. Se anticipa un sentido de conjunto porque leemos el texto con ciertas expectativas sobre un determinado sentido. Después el proyecto se revisa, o proyectos en liza pueden contribuir conjuntamente a una reelaboración hasta fijar con más claridad la unidad de sentido, los preconceptos pueden ser substituidos por conceptos más adecuados. Los esquemas con los que se inicia la comprensión son auténticas hipótesis. No se trata de abordar el texto instalándose en los prejuicios previos, sino de poner a prueba el prejuicio del que partimos (Gadamer 1994e (1959):65 y s.). Por eso una conciencia formada hermenéuticamente debe estar dispuesta a escoger la alteridad del texto. Heidegger reclama que la anticipación y la pre-comprensión se pongan a prueba por las cosas mismas. De esta manera, lejos de tópicos como el de que sólo podemos comprender lo que ya somos, se parte de la pre-comprensión para abrirse a la alteridad.

En el contexto de este artículo, resulta tentador leer lo que antecede o desde la propuesta de Pike, ver el enfoque etic como esa anticipación de sentido que responde a ciertas expectativas, entender los conceptos etic como los preconceptos que han de ser sustituidos por conceptos emic más adecuados. Pero resulta más pertinente leerlo desde la propuesta de Agar, 1992 ( 1982). Al estudiar otras culturas la incomprensión se produce cuando nuestras expectativas no son satisfechas, cuando nuestras pre-concepciones se quiebran. Interpretar sería hacer inteligibles comportamientos que inicialmente no se comprenden. Para indicar como se hace este recorrido Agar recurre a una serie de conceptos tomados de la sociología interpretativa de Schütz. 
Así los conceptos clave para dar cuenta del problema etnográfico, son los de quiebra y esquema corrregible. La quiebra se produce cuando hay una disyunción entre el mundo del etnógrafo y el estudiado, cuando algo de lo que el etnógrafo constata rompe sus expectativas. De ahí que la quiebra exija la corrección de los esquemas del etnógrafo, que tiene que entender el comportamiento de los actores y, en adelante, incluir este tipo de comportamiento en sus esquemas. Para dar cuenta del comportamiento de los actores los conceptos básicos son los que Agar toma de la sociología comprensiva de Schutz:

- meta ("interés a mano")

- repertorio de conocimiento organizado en marcos (frames)

- esquema de acción (orientado hacia la meta)

- foco: selección del "repertorio de conocimiento" (anticipaciones y expectativas) en el que se basa el esquema de acción

- planes: complejos de esquemas de acción

El objetivo de la comprensión es la resolución de quiebras entendiendo el comportamiento inicialmente incomprensible como parte de un plan. El proceso que lleva a la resolución (1992: 131 y 133) consiste en ir aplicando nuevos esquemas, que resultan de la corrección del esquema inicial del etnógrafo, a strips, unidades de comportamiento. Si el nuevo esquema no permite alcanzar la comprensión, si se produce una nueva quiebra, el esquema debe modificarse una y otra vez. En esta modificación progresiva de los esquemas de interpretación el segundo paso, fundamental para la puesta a prueba de la interpretación, consistiría en aplicar el esquema resultante del proceso anterior a otro strip. Se trata de poner a prueba la coherencia de la interpretación. Si el esquema no permite dar cuenta del segundo strip debe ser modificado una y otra vez hasta llegar a un esquema que da cuenta a la vez del strip 1 y del 2, y así con otro strip, y otro, y otro...

En este punto, es llamativa la correspondencia que se puede establecer entre Popper, Hempel, y Lakatos, por una parte, y por otra, Agar y Glazer y Strauss, que le sirven de precedente. ¿Cuando se detiene esta puesta a prueba de la coherencia de la interpretación? Nos encontramos aquí con otra manifestación de los límites del conocimiento. Popper diría que la corroboración no puede ser más que provisional, que una falsación puede producirse posteriormente. Hempel, que las verificaciones no son nunca definitivas; la teoría fundamentada de Glazer y Strauss, que nos detenemos cuando llegamos a un punto de saturación, cuando ya no tenemos a la vista nuevos strips de los que tengamos que dar cuenta. Desde cualquiera de las orientaciones metodológicas nos detenemos por convicción, o por razones pragmáticas, pero nuestros esquemas, relacionales o interpretativos, son siempre provisionales.

Pero, ¿qué ganamos aproximando a Popper a Agar, los programas de investigación de Lakatos y la teoría fundamentada?. Ganamos corroboraciones. Si desde tradiciones tan distintas como el racionalismo crítico, la posición creativa de Glazer y Strauus frente a al enrocamiento de la gran teoría de la sociología norteamericana y la sociología interpretativa se llega a coincidencias, nos encontraríamos en una situación comparable a la que postulaba Pike cuando decía que las aproximaciones preliminares, etic, de distintos etnógrafos podían ser eran más dispares que sus informes finales emic, porque en el primer caso dependerían de los contenidos de sus programas de formación $\mathrm{y}$ en el segundo del descubrimiento, no de la invención, de estructuras preexistentes. En el caso de las metodologías, su aproximación podemos tomarla como indicador de que se aproximan al quehacer de los buenos científicos, de los buenos etnógrafos.

No parece infundado concluir en este punto que, si se entiende por ciencia no un conocimiento que proporcione explicaciones nomológico- deductivas de los fenómenos sino un conocimiento que se dota de mecanismos de puesta a prueba y autocorrección, las interpretaciones pueden ser tan científicas, o serlo tan poco, como las explicaciones causales. 
No parce difícil, tampoco, defender la aplicación de la concepción estructural de las teoría a la Antropología. El conocimiento de una cultura supone la interpretación de significados, el esclarecimiento de razones y motivos de la acción, la identificación de estructuras, procesos y datos contextuales y finalmente, el establecimiento de relaciones entre ellos. Y es en las etnografías donde se construyen los modelos teóricos que articulan los datos, que se supone se han ido poniendo a prueba paso a paso. Queda atrás así aquella secuencia inductivista que pensaba el conocimiento antropológico construido en tres etapas, Etnografía (toma de datos), Etnología (comparación) y Antropología ( teorización).

En cuanto al esbozo de las implicaciones de esta forma de entender la Antropología para la comparación transcultural, volverá a aquella comunicación de 1996 en la que hablé también de un segundo objetivo, reformular la teoría del parentesco haciendo de ella un programa de investigación transcultural de la procreación. Enunciado de forma más modesta, hablaría ahora de la reformulación del dominio de la Antropología del parentesco ${ }^{3}$ y de que el trabajo pendientes es cómo evaluar la fecundidad y comparar los modelos de procreación, adscripción y crianza que estamos construyendo. Por eso uno de los objetivos de este texto fue adelantar qué forma de nuevo procedimiento de comparación, de CM 2 para volver a la cita de Strathern del arranque, corresponde al modo de investigación científica en Antropología aquí propuesto.

Si nos situamos en la perspectiva de la concepción estructural de las teorías, el proceso de desarrollo y puesta prueba de una teoría, supondría ir más allá de las aplicaciones paradigmáticas que se aducen con la teoría y que hacen posible su aceptación para comprobar si se adecua a otras aplicaciones propuestas o que se van proponiendo. Desarrollar una teoría consiste en proponer nuevas aplicaciones, en formular leyes especiales. Podemos encontrar estos procesos en la historia de la Antropología. Sólo pondré dos ejemplos, el desarrollo de la teoría sobre la brujería de 1937 en adelante y el que se hizo de las estructuras semicomplejas de parentesco entre los años 1995 y 2000.

M. Douglas hizo en 1967 un balance de la aplicación del modelo azande a muchas otras sociedades africanas. Y en particular del proceso de discusión sobre la diferencia entre sorcery $\mathrm{y}$ witchcraft porque creencias en un poder aprendido y ejercido de manera consciente no se ajustaban al poder inconsciente e innato del ira wene azande. En los siguientes treinta años, el campo de aplicación del modelo se extendió de las sociedades africanas a sociedades campesinas europeas ( Lisón, Favret-Saada). Pero antes de esta extensión, y en ella, se produjo otra especialización del modelo, a medida que se fue abriendo paso la idea de que los poderes personales especiales que se han conceptualizado como brujería no siempre son antisociales, porque con frecuencia son también los poderes que poseen los grandes terapeutas (Wilson, Middelton, Lison, Favret -Saada, Mallart).

Si pensamos que al menos el primer periodo, el que va de 1937 a 1967, es el periodo álgido de uno de los procedimientos comparativos clásicos, el comparativismo hologeista que desarrolló Murdock primero en Yale, desde el Cross Cultural Survey a los Human Relation Area Files , y después en Pittsburg, a través de la revista Ethnology, vemos como en este período

\footnotetext{
${ }^{3}$ El dominio de la Antropología del Parentesco estaría constituido por los fenómenos socioculturales en tanto, y sólo mientras, los vemos desde la perspectiva de la reproducción del grupo por procreación y crianza de los niños, entendida como la reposición de los seres humanos que lo integran a través de la conceptualización y regulación de la generación, la adscripción de los niños, su cuidado, la atribución de la responsabilidad última de su socialización y enculturación hasta su madurez social y las relaciones que a partir de estos procesos se pueden desarrollar a lo largo de la vida. (González Echevarría, 2005, González Echevarría y otros, s.d., )
} 
coexistieron los dos modos de comparación, aunque el primero no se nombrara porque estaba inscrito en una práctica que más parecía enfatizar la singularidad de cada cultura,

Otro ejemplo claro de etnografía como predicado de cestructura y de su desarrollo es el del modelo de estructura semicompleja de parentesco elaborado por Héritier sobre los samo. Sus rasgos básicos son linajes patrilineales, poliginia, terminologías de tipo omaha, multiplicidad de prohibiciones matrimoniales formuladas en términos de linajes, ausencia de prescripción con tendencia estadística a la endogamia entre comunidades próximas que constituían la unidad política tradicional, androcentrismo. Las relaciones que Héritier establece entre estos rasgos son las siguientes: el sistema de alianzas matrimoniales que hace posible la conjunción entre el gran número de prohibiciones y la endogamia por medio del bouclage, del reanudamiento de alianzas en cuanto cesan las prohibiciones ; la correspondencia entre la estructura de las alianza y la concepción de la persona, de manera que se constata un homomorfismo entre las prohibiciones matrimoniales y las trazas de substancias recibidas de los antepasados que lleva a hablar no de la reanudación de alianzas entre parientes sino de la reconducción al campo de los parientes de los que acaban de dejar de serlo; y la vinculación entre androcentrismo y terminología omaha que Héritier ofrece en una explicación vinculada a la ontología feminista: la madre y la hija del hermano serían designadas por el mismo término porque se inferioriza a la hermana colocándola en la generación de la hija.

Esta estructura semicompleja de parentesco fue contrastada, modificada y extendida a otras sociedades africanas primero, y después a sociedades del resto del mundo, en los seminarios que Héritier impartió en el College de France a finales de los 80 en los que participaron numerosos etnógrafos que aportaron sus propios datos etnográficos al debate y al desarrollo del modelo.

La aplicación a las etnografías de la concepción estructural de las teorías, y su utilización para cancelar la oposición entre Antropología científica y hermenéutica y entre enfoques etic y emic no se justifica porque esta metateoría responda a los desarrollos más recientes de la filosofía de la ciencia, sino porque da cuenta especialmente bien del quehacer de los etnógrafos, desde los estudios sobre brujería a la práctica etnográfica de Geertz en los artículos que integran $L a$ interpretación de las culturas. También se ajusta a representaciones notables del conocimiento sociológico y antropológico. Giddens, en Las nuevas reglas del método sociológico concluía que las tareas primarias de la sociología son la explicación y mediación hermenéutica de formas de vida divergentes dentro de los metalenguajes descriptivos de las ciencias sociales y la explicación de la producción y reproducción de la sociedad como el resultado logrado por la actividad humana (1987 [1967]: 166). Goodenough, en Description and Comparison en Cultural Anthropology, utilizando un concepto restingido de cultura y llamando formas "extraculturales " a la infraestructura ecológico-económica y a la organización social, sostuvo que las teorías antropológicas incluyen relaciones entre formas culturales, relaciones entre formas culturales y formas extra-culturales y relaciones entre formas extra-culturales.

Nos encontramos de nuevo con el criterio de evaluación que Lakatos, 1975b, en su Historia de la Ciencia y de sus reconstrucciones racionales, extendía desde las teorías a las propuestas metateóricas. Si las primeras tienen que confrontarse con los hechos las segundas tiene que confrontarse con el trabajo de los científicos. Como en el caso anterior, las analogías entre pensadores tan distantes como Giddens, Godenough y Geertz y entre desarrollos etnográficos tan dispares como el estudio de la brujería o de los sistemas semicomplejos de alianza matrimonial constituyen para las tesis que sostengo corroboraciones.

Antes de terminar debo reconocer que si no considerara imposible borrar la versión canónica de la dicotomía emic/etic, la que opone los modelos de los antropólogos a los de los nativos, me sentaría tentada como homenaje al interés de los precedentes de Goodenogh y Pike a utilizar bagaje etic para el preliminar del antropólogo, tal como lo analiza Goodenough y comprensión 
emic para lo que fue en Pike: el producto final y más sofisticado de su trabajo. Pero si la primera dificultad es insalvable, otra sería que mis usos divergen de los estos dos autores clásicos tanto que recurrir a sus términos tendría que ir acompañado siempre de numerosas notas a pie de página. Por otra parte, la idea de Pike de alcanzar la estructura de la cultura quedaría subsumida en la propuesta que estoy haciendo. Esta propuesta añade a Pike la búsqueda de causas y razones que pueden relacionarse con intereses, motivaciones, clasificaciones o representaciones, como condiciones de posibilidad o condiciones necesarias y suficientes, o relaciones isomórficas o de otro tipo $\mathrm{y}$, en consecuencia, cumple tanto la exigencia de adecuación a la lógica interna de las culturas estudiadas, que se ha venido discutiendo desde Pike en adelante, como la exigencia de Harris de pertinencia interna a la comunidad antropológica.

La separación entre Antropologías científicas y hermenéuticas con la que yo trabajaba en 1987, al igual que la dicotomía emic/etic a la manera de Harris, son insostenibles porque no se pueden separar comportamientos y significados. Pero además, si la interpretación es el resultado de un proceso científico que va de los preconceptos y de las quiebras a la modificación de los esquemas de los antropólogos. carece de pertinencia.

\section{Bibliografía}

Agar, M. 1982 "Hacia un lenguaje etnográfico", en Reynoso, comp. 1992, El surgimiento de l 'Antropología , Barcelona, Gedisa.

Dilthey, W. 1966(1883) Introducción a las ciencias del espiritu. Madrid. Revista de Occidente.

Douglas, M. (1976). "Brujería: el estado actual de la cuestión”, en M. Gluckman y otros.

Ciencia y brujería. 31-72. Barcelona: Anagrama

Evans-Pritchard,E.E. (1978 [1937]). Brujería, magia y oráculos entre los Azande.

Barcelona: Anagrama

Evans-Pritchard, E.E. (1977 [1940]) Los nuer, Barcelona, Anagrama.

Favret.Saada, J. 1977 Les mots, la mort et les sorts. La sorcellerie dans le Bocage. Paris, Gallimard.

Gadamer, H.G,1993(1960) Verdad y Método I. Salamanca. Sígueme.

1994(1986) Verdad y Método, II. Salamanca. Sígueme.

1994a (1953) “La verdad en las ciencias del espíritu”, en Gadamer, 1994: 43-49.

1994b(1957) “¿Qué es la verdad? en Gadamer, 1994: 51-60.

1994 c(1985) “Entre fenomenología y dialéctica. Intento de una autocrítica”, en Gadamer, 1994: 11-29.

1994d (1977)“Auto-representación de Hans Goerg Gadamer”, en Gadamer, 1994: 375-

402.

1994e (1959) "Sobre el círculo de la comprensión”, en Gadamer, 1994: 63-70.

Douglas, M. 1976. "Brujería: el estado actual de la cuestión", en M. Gluckman y

otros. Ciencia y brujería. 31-72. Barcelona: Anagrama

Geertz, C. 1987 (1973) La interpretación de las culturas. México. Gedisa.

Gibbons et alli. 1994 The New Production of Knowledge: The dinamics of Science and

Research in Contemprary Societies, London.Sage. Cit.por Strathern, 2002

Giddens, A. (1987 (1967)) Las nuevas reglas del método sociológico. Una crítica positiva de la sociología interpretativa, Buenos Aires, Amorrortu

Glaser, B.G. y Strauuss, A.L1967 The Discovery of Grounded Theory: strategies for qualitative research. New York: Aldine.

González Echevarría, A.1987 La construcción teórica en antropología. Barcelona. Anthropos 
1996 "Conceptos interpretativos y conceptos teóricos", en González Echevarría, A., ed. Simposium sobre 'Epistemología y Antropología'. Actas del VI Congreso de Antropología del Estado Español: 115-131

2003 Crítica de la singularidad cultural Barcelona/ México D.F. Anthropos/ Universidad Autónoma Metropolitana.

2005 "Informe sobre el estado del desarrollo de la Antropología de la procreación", Perifèria, n. 3, www.periferia.name

2009. La dicotomía emic/etic. Historia de una confusión, Barcelona, Anthropos

González Echevarría y otros, s.d., "La Antropología del Parentesco como estudio transcultural de la reproducción de los grupos humanos: Modelos socioculturales de procreación, circulación y crianza de niños", art.en evaluación.

Goodenough, W.H 1956b "Componential Analysis and the study of Meaning". Language vol 32:195-216.

1956a "Residence Rules" Southwestern Journal of Anthropology,11: 22-37.

1970 Description and Comparation in Cultural Anthropology. Cambridge: Cambridge U.P.

Hammel, E.A. 1964 "Further comments on componential analysis", American Anthropologist, 66: 1176-1171. Reproducido en N. Graburn, Readings in Kinship and Social Structure. New York. Harper and Row, 1971:285-288.

Harris, M. 1964 The Nature of Cultural Things. New York. Rondon House.

1971 Culture, Man and Nature. An Introduction to General Anthropology. New York.Thomas Y. Crowell $\mathrm{C}^{\circ}$.

1978 (1968) El desarrollo de la teoría antropológica. Madrid. s.XXI.

Héritier, F. 1981, L'exercice de la parenté. París. Editions du Seuil.

Héritier- Augé, F. y Copet-Rougier, E. eds (1990). Les complexites de L'Alliance. Vol I.Les systèmes semicomplexes. París, Éditions des Archives Contemporaires

Kuhn, T.S. (1971 [1969]) “Posdata: 1969”, en La estructura de las revoluciones científicas. México. F.C.E.

Lakatos, I. 1975a "La falsación y la metodología de los programas de investigación científica", en I. Lakatos y A. Musgrave, eds. 1975: 203-343.

1975b "La historia de la ciencia y sus reconstrucciones racionales", en I. Lakatos y A.

Musgrave, eds. 1975.

Lakatos, I.y Musgrave, eds. 1975 La crítica y el desarrollo del conocimiento Barcelona. Grijalbo.

Lisón, C.1979 Brujería, estructural social y simbolismo en Galicia Madrid, Akal.

Mallart, L. 2009 El sistema medic d'una societat africana. Els evuzok de Camerun. 2 vols.

Barcelona, Institut d'Estudis Catalans.

Middelton, J. 1960 Lugbara Religion. Londres. Oxford U.

Nowotny , H. , Scott, P. , Gibbons, M. 2001, Re-Thinking Science: Knwledge and the Public in an Age of Uncertainty, Oxford Polity: Press. Cit.por Strathern, 2002.

Pike, K.L. 1954 Language in relation to a unified theory of the structure of human behavior. Preliminary Edition. Volumen 1. (Capítulos 1-7). Glendale. Calif. Summer Institute of Linguistic.

1955 Language in relation to a unified theory of the structure of human behavior. Preliminary Edition. Volumen 2 (capítulos 8-9) Glendale. Calif. Summer Institute of Linguistic.

1960 Language in Relation to a unified theory of the structure of human behavior. Preliminary Edition. Volumen 3 (capítulos 11-17). Glendale. Calif. Summer Institute of Linguistic.

1971 Language in Relation to a Unified Theory of the Structure of Human Behavior. $2^{\mathrm{a}}$ impresión de la $2^{a}$ edición revisada de 1967. La Haya, Mouton Saussure, F. de 1965 (1915) Curso de Lingüistica General. Buenos Aires. Losada. 
Schütz, A. 1972 (1932) Fenomenología del mundo social. Introducción a la sociología comprensiva. Buenos Aires. Paidós.

Suippes, S.1962. "Models of Data", en Nagel, E., Suppe, F., Tarks, A. (eds.) Logic,

Methodology and Philosophy of Science, Proceedings of the 1960 International Congress (252261). Stanford: Standford U.P.

(1966). "Wath is a Scientific Theory?" en Morgenbesser, S. Philosophy of Science Today (5567). Nueva York: Basic Books.

Strathern, M. "Foreword” en Gringrich , A. y Fox , R.G. eds 2002 ,Anthropology, by Comparison, Routledge : XIII-XVII

Wallace, A.F. C. y Atkins, J. 1960 "The meaning of kinship Terms". American Anthropologist, 62: $58-80$.

Wilson. M. (1951) "Witch belief and social structure" American Journal of Sociology, vol. LVI, 4, pp-307-313.

Winch, P. [1958] The Idea of a Social Science and its Relations to Philosophy. London. 